Sharif University of Technology
Scientia Iranica
Transactions E: Industrial Engineering
SCIENTIA

\title{
Efficient estimation of population median using supplementary variable
}

\author{
S. Muneer ${ }^{a, *}$, A. Khalil ${ }^{a}$, J. Shabbir ${ }^{b}$, and Gh. Narjis ${ }^{b}$ \\ a. Department of Statistics, University of Peshawar, Peshawar, Pakistan. \\ b. Department of Statistics, Quaid-i-Azam University, Islamabad, Pakistan. \\ Received 5 February 2019; received in revised form 22 January 2020; accepted 11 May 2020
}

\section{KEYWORDS}

Bias;

Efficiency;

Supplementary

variable;

Median.

\begin{abstract}
The present study aims to introduce an exponential ratio-cum-product type class of estimators for population median under simple random sampling scheme using the supplementary variable. Expressions for the bias and Mean Square Error (MSE) were obtained up to the first order of approximation. The proposed class of estimators was more efficient than all other considered estimators under certain conditions. Four real datasets and simulation studies were carried out to assess the performances of these estimators. Both numerical and simulation studies demonstrated that the proposed class of estimators outperformed the other considered estimators.
\end{abstract}

(C) 2022 Sharif University of Technology. All rights reserved.

\section{Introduction}

In situations where the supplementary variable is correlated with the study variable, application of the appropriate supplementary variable may result in a considerable reduction in Mean Squared Error (MSE) of the regression, ratio, and product estimators. The main objective of a number of statistical analyses is to estimate the location parameter for the desired distribution, which describes the data in good manners. In case the entire data are homogeneous, the sample mean performs better. There are situations in survey sampling when the study variable indicates an extremely skewed distribution such as expenditure, taxes, income, production, and consumption. In addition, if some of the observations in the data greatly differ from the others, the sample mean performs very poorly, and the results are either under- or over-estimated. To handle such situations, median is used as an alternative

\footnotetext{
*. Corresponding author. Tel.: +923145811676 E-mail address: sirajmuneer1@gmail.com (S. Muneer)
}

measure of location since it performs better in case of skewed variable and gives an efficient estimation of the population parameter. A considerably rich literature is available on the estimation of the mean and total; however, comparatively less efforts have been made to propose efficient estimators of the population median $\left(M_{y}\right)$. Gross [1] was the first to have proposed a weighted estimator without a supplementary variable for population median $\left(M_{y}\right)$ and derived its asymptotic distribution. Kuk and Mak [2] suggested the classical ratio method for population median using supplementary information. Singh et al. [3] presented a class of generalized ratio type estimators for population median using the supplementary variable. Singh et al. [4,5] introduced certain estimators for population median using known parameters of the supplementary variables. Singh et al. [6] suggested chain ratio and regression estimators for population median. Gupta et al. [7] recommended a number of difference-type estimators for population median using the supplementary variables. Other important contributions in this area are the studies conducted by Aladag and Cingi [8], Singh and Solanki [9], Jhajj et al. [10], Sharma and Singh [11], Aladag and Cingi [12], Shabbir 
and Gupta [13,14], Solanki and Singh [15], Enang et al. [16], Jhajj et al. [17], Koyuncu [18], Irfan et al. [19], Yadav et al. [20], Bandyopadhyay et el. [21], Javed et al. [22], and Baig et al. [23]. These researchers proposed a large number of estimators to estimate the population parameters under different situations using the supplementary variables.

In the present study, a new class of ratio-cumproduct type exponential estimators for population median $\left(M_{y}\right)$ was proposed under Simple Random Sampling (SRS) scheme using the supplementary variable. This research primarily aims to propose an efficient class of estimators to increase the precision of the median estimators.

Consider a finite population $U=\left\{U_{1}, U_{2}\right.$, $\left.\cdots, U_{N}\right\}$ with $N$ units. Let $\left(y_{i}, x_{i}\right)$ be the $i$ th values of the survey variable $y$ and supplementary variable $x$, respectively. A sample of size $n$ was selected from this population using Simple Random Sample Without Replacement (SRSWOR) scheme. Let $\widehat{M}_{y}$ and $\widehat{M}_{x}$ be the sample medians corresponding to the population medians $M_{y}$ and $M_{x}$, respectively. Let $f_{y}\left(M_{y}\right)$ and $f_{x}\left(M_{x}\right)$ be the probability density functions of $M_{y}$ and $M_{x}$, respectively. Let $\rho_{\left(\widehat{M}_{y}, \widehat{M}_{x}\right)}=\rho_{y x}=4 P_{11}(y, x)-$ 1 be the coefficient of correlation between $\widehat{M}_{y}$ and $\widehat{M}_{x}$, where $P_{11}$ indicates the fraction of values in the population with $Y \leq M_{Y}$ and $X \leq M_{X}$. It is assumed that $(y, x)$ follows a continuous distribution with $f_{y}\left(M_{y}\right)$ and $f_{x}\left(M_{x}\right)$ as their marginal densities.

To get the bias and MSE of the estimators, the following relative error terms are used.

Let:

$$
\zeta_{0}=\left(\frac{\widehat{M}_{y}-M_{y}}{M_{y}}\right) \quad \text { and } \quad \zeta_{1}=\left(\frac{\widehat{M}_{x}-M_{x}}{M_{x}}\right),
$$

such that:

$$
\begin{aligned}
& E\left(\zeta_{j}\right)=0, \quad(j=0,1), \quad E\left(\zeta_{0}^{2}\right)=\lambda \psi_{M_{y}}^{2}, \\
& E\left(\zeta_{1}^{2}\right)=\lambda \psi_{M_{x}}^{2}, \quad E\left(\zeta_{0} \zeta_{1}\right)=\lambda \psi_{M_{y x}},
\end{aligned}
$$

where:

$$
\begin{aligned}
& \psi_{M_{y}}=\frac{1}{\left[M_{y} f_{y}\left(M_{y}\right)\right]}, \quad \psi_{M_{x}}=\frac{1}{\left[M_{x} f_{x}\left(M_{x}\right)\right]}, \\
& \psi_{M_{y x}}=\rho_{y x} \psi_{M_{y}} \psi_{M_{x}},
\end{aligned}
$$

and:

$$
\lambda=\frac{1}{4}\left(\frac{1}{n}-\frac{1}{N}\right) .
$$

\section{Estimators in literature}

This section discusses the following usual median per unit estimator and some existing estimators of the population median $M_{y}$ : (i) The most common estimator of the median $M_{y}$ is sample median estimator $\widehat{M}_{y}$ whose variance is calculated by:

$$
V\left(\widehat{M}_{y}\right)=\lambda M_{y}^{2} \psi_{M_{y}}^{2}
$$

(ii) The usual ratio estimator of the median suggested by Kuk and Mak [2] is:

$$
\widehat{M}_{R}=\widehat{M}_{y}\left(\frac{M_{x}}{\widehat{M}_{x}}\right) \text {. }
$$

The bias and MSE of $\widehat{M}_{R}$ are given by:

$$
B\left(\widehat{M}_{R}\right) \cong \lambda M_{y}\left(\psi_{M_{x}}^{2}-\psi_{M_{y x}}\right)
$$

and:

$$
\operatorname{MSE}\left(\widehat{M}_{R}\right) \cong \lambda M_{y}^{2}\left(\psi_{M_{y}}^{2}+\psi_{M_{x}}^{2}-2 \psi_{M_{y x}}\right)
$$

(iii) The usual exponential estimator is:

$$
\widehat{M}_{\operatorname{Exp}}=\widehat{M}_{y} \exp \left(\frac{M_{x}-\widehat{M}_{x}}{M_{x}+\widehat{M}_{x}}\right) .
$$

The bias and MSE of $\widehat{M}_{\mathrm{Exp} \mid}$, are given by:

$$
B\left(\widehat{M}_{\operatorname{Exp}}\right) \cong \lambda M_{y}\left(\frac{3}{8} \psi_{M_{x}}^{2}-\frac{1}{2} \psi_{M_{y x}}\right)
$$

and:

$$
\operatorname{MSE}\left(\widehat{M}_{\mathrm{Exp}}\right) \cong \lambda M_{y}^{2}\left(\psi_{M_{y}}^{2}+\frac{1}{4} \psi_{M_{x}}^{2}-\psi_{M_{y x}}\right)
$$

(iv) Singh and Solanki [9] proposed the following estimators for $M_{y}$ as:

$$
\begin{aligned}
& \widehat{M}_{S S 1}=k_{1} \widehat{M}_{y}+\left(1-k_{1}\right)\left(M_{x}-\widehat{M}_{x}\right) \\
& \widehat{M}_{S S 2}=k_{2} \widehat{M}_{y}+k_{3}\left(M_{x}-\widehat{M}_{x}\right)
\end{aligned}
$$

where $k_{1}, k_{2}$, and $k_{3}$ are the constants.

To the first degree of approximation, the biases and minimum MSEs of $\widehat{M}_{S S i}(i=1,2)$ are given by:

$$
\begin{aligned}
& B\left(\widehat{M}_{S S 1}\right) \cong\left(k_{1}-1\right) M_{y}, \\
& B\left(\widehat{M}_{S S 2}\right) \cong\left(k_{2}-1\right) M_{y}, \\
& M S E\left(\widehat{M}_{S S 1}\right)_{\min } \cong M_{y}^{2}\left[1+\frac{M_{x}^{2}}{M_{y}^{2}} \lambda \psi_{M_{x}}^{2}\right. \\
& \left.\quad \frac{\left(1+\frac{M_{x}^{2}}{M_{y}^{2}} \lambda \psi_{M_{x}}^{2}+\frac{M_{x}}{M_{y}} \lambda \psi_{M_{y x}}\right)^{2}}{1+\lambda \psi_{M_{y}}^{2}+\frac{M_{x}^{2}}{M_{y}^{2}} \lambda \psi_{M_{x}}^{2}+2 \frac{M_{x}}{M_{y}} \lambda \psi_{M_{y x}}}\right],
\end{aligned}
$$




$$
\operatorname{MSE}\left(\widehat{M}_{S S 2}\right)_{\min } \cong \frac{\lambda M_{y}^{2} \psi_{M_{y}}^{2}\left(1-\rho_{y x}^{2}\right)}{1+\lambda \psi_{M_{y}}^{2}\left(1-\rho_{y x}^{2}\right)}
$$

The optimal values of $k_{j}(j=1,2,3)$ are:

$$
\begin{aligned}
& k_{1(\mathrm{opt})}=\frac{M_{y}^{2}+M_{x}^{2} \lambda \psi_{M_{x}}^{2}-M_{y} M_{x} \lambda \psi_{M_{y x}}}{M_{y}^{2}+M_{y}^{2} \lambda \psi_{M_{y}}^{2}+M_{x}^{2} \lambda \psi_{M_{x}}^{2}}, \\
& k_{2(\mathrm{opt})}=\frac{1}{1+\lambda \psi_{M_{y}}^{2}\left(1-\rho_{y x}^{2}\right)}, \\
& k_{3(\mathrm{opt})}=\frac{M_{y}}{M_{x}}\left[\frac{\frac{\rho_{y x} \psi_{M_{y}}}{\psi_{M_{x}}}}{1+\lambda \psi_{M_{y}}^{2}\left(1-\rho_{y x}^{2}\right)}\right] .
\end{aligned}
$$

(v) The difference estimator of the median is calculated in the following:

$$
\widehat{M}_{D}=\widehat{M}_{y}-k_{4}\left(\widehat{M}_{x}-M_{X}\right)
$$

where $k_{4}$ is the unknown constant whose value is to be found.

The minimum MSE of $\widehat{M}_{D}$ can be obtained through the following equation:

$$
\operatorname{MSE}\left(\widehat{M}_{D}\right)_{\min } \cong \lambda M_{y}^{2} \psi_{M_{y}}^{2}\left(1-\rho_{y x}^{2}\right) .
$$

The optimum value of $k_{4}$ is:

$$
k_{4(\mathrm{opt})}=\frac{f_{x}\left(M_{x}\right)}{f_{y}\left(M_{y}\right)} \rho_{y x} .
$$

(vi) Solanki and Singh [15] proposed a generalized class of estimators for median as follows:

$\widehat{M}_{S S 3}=\widehat{M}_{y}\left[k_{5}\left(\frac{c M_{X}+d}{c \widehat{M}_{x}+d}\right)+k_{6}\left(\frac{c \widehat{M}_{x}+d}{c M_{x}+d}\right)\right]$

where $k_{5}$ and $k_{6}$ are the unknown constants; $c$ and $d$ the known population parameters of the supplementary variable.

The bias and minimum MSE of $\widehat{M}_{S S 3}$, at $(c, d)=(1,0)$, are given by Eqs. (17) and (18) as shown in Box I. The optimum values of $k_{5}$ and $k_{6}$ are obtained by the equations shown in Box II.

(vii) Shabbir and Gupta [14] suggested the following difference-type estimators of the median as follows:

$$
\begin{aligned}
\widehat{M}_{D 1}= & {\left[k_{7} \widehat{M}_{y}+k_{8}\left(M_{x}-\widehat{M}_{x}\right)\right]\left(\frac{M_{x}}{\widehat{M}_{x}}\right), } \\
\widehat{M}_{D 2}= & {\left[k_{9} \widehat{M}_{y}+k_{10}\left(M_{x}-\widehat{M}_{x}\right)\right] } \\
& \exp \left(\frac{M_{x}-\widehat{M}_{x}}{M_{x}+\widehat{M}_{x}}\right),
\end{aligned}
$$

where $k_{j}(j=7,8,9,10)$ are the constants.

The biases and minimum MSEs of $\widehat{M}_{D i}(i=$ $1,2)$ are given by:

$$
B\left(\widehat{M}_{S S 3}\right) \cong M_{y}\left[k_{5}\left(1-\lambda \psi_{M_{y x}}+\lambda \psi_{M_{x}}^{2}\right)+k_{6}\left(1+\lambda \psi_{M_{y x}}\right)-1\right]
$$

and:

$$
M S E\left(\widehat{M}_{S S 3}\right)_{\min } \cong \frac{\lambda M_{y}^{2}\left(\begin{array}{c}
\lambda \psi_{M_{x}}^{6}+\lambda \psi_{M_{y}}^{2} \psi_{M_{x}}^{4}+2 \lambda \psi_{M_{x}}^{4} \psi_{M_{y x}}-4 \lambda \psi_{M_{y}}^{2} \psi_{M_{x}}^{2} \psi_{M_{y x}}-4 \lambda \psi_{M_{x}}^{2} \psi_{M_{y x}}^{2} \\
+4 \lambda \psi_{M_{y}}^{2} \psi_{M_{y x}}^{2}-4 \psi_{M_{y}}^{2} \psi_{M_{x}}^{2}+4 \psi_{M_{y x}}^{2}
\end{array}\right)}{16 \lambda \psi_{M_{y x}}^{2}-4 \psi_{M_{x}}^{2}-8 \lambda \psi_{M_{x}}^{2} \psi_{M_{y x}}-4 \lambda \psi_{M_{y}}^{2} \psi_{M_{x}}^{2}-3 \lambda \psi_{M_{x}}^{4}}
$$

Box I

$$
k_{5(\mathrm{opt})}=\left(\frac{\lambda \psi_{M_{y}}^{2} \psi_{M_{x}}^{2}-3 \lambda \psi_{M_{x}}^{2} \psi_{M_{y x}}-2 \lambda \psi_{M_{y}}^{2} \psi_{M_{y x}}+4 \lambda \psi_{M_{y x}}^{2}-2 \psi_{M_{x}}^{2}+2 \psi_{M_{y x}}}{16 \lambda \psi_{M_{y x}}^{2}-4 \psi_{M_{x}}^{2}-8 \lambda \psi_{M_{x}}^{2} \psi_{M_{y x}}-4 \lambda \psi_{M_{y}}^{2} \psi_{M_{x}}^{2}-3 \lambda \psi_{M_{x}}^{4}}\right)
$$

and:

$$
k_{6(\mathrm{opt})}=\left(\frac{\lambda \psi_{M_{x}}^{4}+\lambda \psi_{M_{y}}^{2} \psi_{M_{x}}^{2}+3 \lambda \psi_{M_{x}}^{2} \psi_{M_{y x}}-2 \lambda \psi_{M_{y}}^{2} \psi_{M_{y x}}-4 \lambda \psi_{M_{y x}}^{2}+2 \psi_{M_{x}}^{2}+2 \psi_{M_{y x}}}{16 \lambda \psi_{M_{y x}}^{2}-4 \psi_{M_{x}}^{2}-8 \lambda \psi_{M_{x}}^{2} \psi_{M_{y x}}-4 \lambda \psi_{M_{y}}^{2} \psi_{M_{x}}^{2}-3 \lambda \psi_{M_{x}}^{4}}\right) .
$$




$$
\begin{gathered}
B\left(\widehat{M}_{D 1}\right) \cong\left(k_{7}-1\right) M_{y} \\
+k_{8} \lambda M_{y}\left(\psi_{M_{x}}^{2}-\psi_{M_{y x}}\right)+k_{8} \lambda M_{x} \psi_{M_{x}}^{2}, \\
B\left(\widehat{M}_{D 2}\right) \cong\left(k_{9}-1\right) M_{y} \\
+k_{9} \lambda M_{y}\left(\frac{3}{8} \psi_{M_{x}}^{2}-\frac{1}{2} \psi_{M_{y x}}\right) \\
+\frac{1}{2} k_{10} \lambda M_{x} \psi_{M_{x}}^{2}, \\
M S E\left(\widehat{M}_{D 1}\right)_{\min } \cong M_{y}^{2} \\
\quad\left[\frac{\left(1-\lambda \psi_{M_{x}}^{2}\right) \lambda \psi_{M_{y}}^{2}\left(1-\rho_{y x}^{2}\right)}{\left(1-\lambda \psi_{M_{x}}^{2}\right)+\lambda \psi_{M_{y}}^{2}\left(1-\rho_{y x}^{2}\right)}\right] \\
M S E\left(\widehat{M}_{D 2}\right)_{\min } \cong M_{y}^{2}\left[1-\frac{1}{4} \lambda \psi_{M_{x}}^{2}\right. \\
\left.\quad-\frac{\left(1-\frac{1}{8} \lambda \psi_{M_{x}}^{2}\right)^{2}}{1+\lambda \psi_{M_{y}}^{2}\left(1-\rho_{y x}^{2}\right)}\right] .
\end{gathered}
$$

The optimal values of $k_{j}(j=7,8,9,10)$ are:

$$
\begin{aligned}
k_{7(\mathrm{opt})} & =\frac{\left(1-\lambda \psi_{M_{x}}^{2}\right)}{1-\lambda \psi_{M_{x}}^{2}+\lambda \psi_{M_{y}}^{2}\left(1-\rho_{y x}^{2}\right)}, \\
k_{8(\mathrm{opt})} & =\frac{M_{y}}{M_{x}}\left[1+k_{7(\mathrm{opt})}\left(\frac{\rho_{y x} \psi_{M_{y}}}{\psi_{M_{x}}}-2\right)\right], \\
k_{9(\mathrm{opt})} & =\frac{1-\frac{1}{8} \lambda \psi_{M_{x}}^{2}}{1+\lambda \psi_{M_{y}}^{2}\left(1-\rho_{y x}^{2}\right)},
\end{aligned}
$$

and:

$$
k_{10(\mathrm{opt})}=\frac{M_{y}}{M_{x}}\left[\frac{1}{2}+k_{9(\mathrm{opt})}\left(\frac{\rho_{y x} \psi_{M_{y}}}{\psi_{M_{x}}}-1\right)\right] .
$$

\section{The proposed estimator}

Motivated by Solanki and Singh [15] and Muneer et al. [24], we proposed the following class of exponential ratio-cum-product type estimators of population median $M_{y}$ as follows:

$$
\begin{aligned}
\widehat{M}_{\text {Prop }}= & \widehat{M}_{y}\left[k_{11}\left(\frac{M_{x}}{\widehat{M}_{x}}\right)+k_{12}\left(\frac{\widehat{M}_{x}}{M_{x}}\right)\right] \\
& {\left[\alpha \exp \left(\frac{M_{x}-\widehat{M}_{x}}{M_{x}+\widehat{M}_{x}}\right)\right.} \\
& \left.+(1-\alpha) \exp \left(\frac{\widehat{M}_{x}-M_{x}}{M_{x}+\widehat{M}_{x}}\right)\right],
\end{aligned}
$$

where $\left(k_{j}, j=11,12\right)$ are the unknown constants whose values are to be determined and $\alpha$ is the known constant that takes values of $(0,1)$. By substituting the values of $\alpha$ into Eq. (25), we get the following two estimators:

(i) Put $\alpha=0$; then, the proposed estimator is reduced to:

$$
\begin{aligned}
\left.\widehat{M}_{\mathrm{Prop}(\alpha=0}\right)= & \widehat{M}_{y}\left[k_{11}\left(\frac{M_{x}}{\widehat{M}_{x}}\right)+k_{12}\left(\frac{\widehat{M}_{x}}{M_{x}}\right)\right] \\
& \exp \left(\frac{\widehat{M}_{x}-M_{x}}{M_{x}+\widehat{M}_{x}}\right) .
\end{aligned}
$$

(ii) Put $\alpha=1$, and the proposed estimator is reduced to:

$$
\begin{aligned}
\widehat{M}_{\mathrm{Prop}(\alpha=1)}= & \widehat{M}_{y}\left[k_{11}\left(\frac{M_{x}}{\widehat{M}_{x}}\right)+k_{12}\left(\frac{\widehat{M}_{x}}{M_{x}}\right)\right] \\
& \exp \left(\frac{M_{x}-\widehat{M}_{x}}{M_{x}+\widehat{M}_{x}}\right) .
\end{aligned}
$$

By expressing $\widehat{M}_{\text {Prop }}$ in Eq. (25) in terms of $\left(\zeta_{j}, j=\right.$ $0,1)$ up to the first order of approximation, we get:

$$
\begin{aligned}
& \widehat{M}_{\text {Prop }} \cong M_{y}\left(1+\zeta_{0}\right)\left[k_{11}\left(1-\zeta_{1}+\zeta_{1}^{2}\right)\right. \\
& \left.\quad+k_{12}\left(1+\zeta_{1}\right)\right]\left[1+(1-2 \alpha) \frac{\zeta_{1}}{2}-(1 !-4 \alpha) \frac{\zeta_{1}^{2}}{8}\right] .
\end{aligned}
$$

Keeping $\zeta^{\prime} s$ up to power two and expanding Eq. (28), we have:

$$
\begin{aligned}
\widehat{M}_{\text {Prop }} & -M_{y} \cong M_{y}\left[k _ { 1 1 } \left\{1+\zeta_{0}-\left(\alpha+\frac{1}{2}\right) \zeta_{1}\right.\right. \\
& \left.-\left(\alpha+\frac{1}{2}\right) \zeta_{0} \zeta_{1}+\left(\frac{3}{8}+\frac{3 \alpha}{2}\right) \zeta_{1}^{2}\right\} \\
& +k_{12}\left\{1+\zeta_{0}+\left(\frac{3}{2}-\alpha\right) \zeta_{1}+\left(\frac{3}{2}-\alpha\right) \zeta_{0} \zeta_{1}\right. \\
& \left.\left.+\left(\frac{3}{8}-\frac{\alpha}{2}\right) \zeta_{1}^{2}\right\}-1\right] .
\end{aligned}
$$

Solving Eq. (29), the bias and MSE of $\widehat{M}_{\text {Prop }}$ are:

$$
\begin{aligned}
\operatorname{Bias}\left(\widehat{M}_{\text {Prop }}\right) \cong M_{y}\left[\left(k_{11}+k_{12}-1\right)\right. \\
+k_{11} \lambda\left\{\left(\frac{3}{8}+\frac{3 \alpha}{2}\right) \psi_{M_{x}}^{2}-\left(\alpha+\frac{1}{2}\right) \psi_{M_{y x}}\right\} \\
\left.\quad+k_{12} \lambda\left\{\left(\frac{3}{2}-\alpha\right) \psi_{M_{y x}}+\left(\frac{3}{8}-\frac{\alpha}{2}\right) \psi_{M_{x}}^{2}\right\}\right],
\end{aligned}
$$

and: 


$$
\begin{aligned}
M S E\left(\widehat{M}_{\text {Prop }}\right) \cong M_{y}^{2}\left[1+k_{11}^{2}\left\{1+\lambda \psi_{M_{y}}^{2}\right.\right. \\
\left.+\left(\alpha^{2}+4 \alpha+1\right) \lambda \psi_{M_{x}}^{2}-(4 \alpha+2) \lambda \psi_{M_{y x}}\right\} \\
+k_{12}^{2}\left\{1+\lambda \psi_{M_{y}}^{2}+\left(\alpha^{2}-4 \alpha+3\right) \lambda \psi_{M_{x}}^{2}\right. \\
\left.+(6-4 \alpha) \lambda \psi_{M_{y x}}\right\}+2 k_{11} k_{12}\left\{1+\lambda \psi_{M_{y}}^{2}\right. \\
\left.+(2-4 \alpha) \lambda \psi_{M_{y x}}+\alpha^{2} \lambda \psi_{M_{x}}^{2}\right\} \\
\quad-2 k_{11}\left\{1+\left(\frac{3}{8}+\frac{3 \alpha}{2}\right) \lambda \psi_{M_{x}}^{2}\right. \\
\left.+\left(\alpha+\frac{1}{2}\right) \lambda \psi_{M_{y x}}\right\}-2 k_{12}\{1 \\
\left.\left.+\left(\frac{3}{8}-\frac{\alpha}{2}\right) \lambda \psi_{M_{x}}^{2}+\left(\frac{3}{2}-\alpha\right) \lambda \psi_{M_{y x}}\right\}\right]
\end{aligned}
$$

or:

$$
\begin{gathered}
\operatorname{MSE}\left(\widehat{M}_{\text {Prop }}\right) \cong M_{y}^{2}\left[1+k_{11}^{2} A_{1}+k_{12}^{2} A_{2}\right. \\
\left.\quad+2 k_{11} k_{12} A_{3}-2 k_{11} A_{4}-2 k_{12} A_{5}\right],
\end{gathered}
$$

where:

$$
\begin{aligned}
A_{1}= & +\lambda \psi_{M_{y}}^{2}+\left(\alpha^{2}+4 \alpha+1\right) \lambda \psi_{M_{x}}^{2} \\
& -(4 \alpha+2) \lambda \psi_{M_{y x}} \\
A_{2}= & +\lambda \psi_{M_{y}}^{2}+\left(\alpha^{2}-4 \alpha+3\right) \lambda \psi_{M_{x}}^{2} \\
& +(6-4 \alpha) \lambda \psi_{M_{y x}}, \\
A_{3}= & +\lambda \psi_{M_{y}}^{2}+(2-4 \alpha) \lambda \psi_{M_{y x}}+\alpha^{2} \lambda \psi_{M_{x}}^{2}, \\
A_{4}= & +\left(\frac{3}{8}+\frac{3 \alpha}{2}\right) \lambda \psi_{M_{x}}^{2}-\left(\alpha+\frac{1}{2}\right) \lambda \psi_{M_{y x}}, \\
A_{5}= & +\left(\frac{3}{8}-\frac{\alpha}{2}\right) \lambda \psi_{M_{x}}^{2}+\left(\frac{3}{2}-\alpha\right) \lambda \psi_{M_{y x}} .
\end{aligned}
$$

Differentiate Eq. (31) with respect to $k_{11}$ and $k_{12}$ so that the optimal values of $k_{11}$ and $k_{12}$ can be obtained as follows:

$$
k_{11(\mathrm{opt})}=\left(\frac{A_{2} A_{4}-A_{3} A_{5}}{A_{1} A_{2}-A_{3}^{2}}\right) \text {, }
$$

and:

$$
k_{12(\mathrm{opt})}=\left(\frac{A_{1} A_{5}-A_{3} A_{4}}{A_{1} A_{2}-A_{3}^{2}}\right) .
$$

Substitute the optimal values of $k_{11}$ and $k_{12}$ into
Eq. (31); then, the minimum MSE of $\widehat{M}_{\text {Prop }}$ can be obtained by:

$$
\begin{aligned}
M S E & \left(\widehat{M}_{\text {Prop }}\right)_{\min } \cong M_{y}^{2} \\
& \left(1-\frac{A_{1} A_{5}^{2}-2 A_{3} A_{4} A_{5}+A_{2} A_{4}^{2}}{A_{1} A_{2}-A_{3}^{2}}\right) .
\end{aligned}
$$

\section{Theoretical comparison}

Here, the MSE of the proposed estimator $\widehat{M}_{\text {Prop }}$ is compared with the MSEs of the considered estimators to derive the optimal conditions:

Condition (i): By Eqs. (1) and (32):

$$
V\left(\widehat{M}_{y}\right)-M S E\left(\widehat{M}_{\text {Prop }}\right)_{\min }>0
$$

if:

$$
M_{y}^{2}\left(\left(\lambda \psi_{M_{y}}^{2}-1\right) \theta_{2}+\theta_{1}\right)>0
$$

where:

$$
\theta_{1}=A_{1} A_{5}^{2}-2 A_{3} A_{4} A_{5}+A_{2} A_{4}^{2},
$$

and:

$$
\theta_{2}=A_{1} A_{2}-A_{3}^{2}
$$

Condition (ii): By Eqs. (4) and (32),

$$
M S E\left(\widehat{M}_{R}\right)-\operatorname{MSE}\left(\widehat{M}_{\mathrm{Prop}}\right)_{\min }>0
$$

if:

$$
M_{y}^{2}\left(\left(\lambda \psi_{M_{y}}^{2}+\lambda \psi_{M_{x}}^{2}-2 \lambda \psi_{M_{y x}}-1\right) \theta_{2}+\theta_{1}\right)>0 .
$$

Condition (iii): By Eqs. (7) and (32), we have:

$$
M S E\left(\widehat{M}_{\mathrm{Exp}}\right)-M S E\left(\widehat{M}_{\text {Prop }}\right)_{\min }>0,
$$

if:

$$
M_{y}^{2}\left(\left(\lambda \psi_{M_{y}}^{2}+\frac{1}{4} \lambda \psi_{M_{x}}^{2}-\lambda \psi_{M_{y x}}-1\right) \theta_{2}+\theta_{1}\right)>0 .
$$

Condition (iv): By Eqs. (12) and (32), we have:

$$
M S E\left(\widehat{M}_{S S 1}\right)_{\min }-M S E\left(\widehat{M}_{\mathrm{Prop}}\right)_{\min }>0
$$

if:

$$
\begin{aligned}
& M_{y}^{2}\left(\frac{M_{x}^{2}}{M_{y}^{2}} \lambda \psi_{M_{x}}^{2}-\frac{\left(1+\frac{M_{x}^{2}}{M_{y}^{2}} \lambda \psi_{M_{x}}^{2}+\frac{M_{x}}{M_{y}} \lambda \psi_{M_{y x}}\right)^{2}}{1+\lambda \psi_{M_{y}}^{2}+\frac{M_{x}^{2}}{M_{y}^{2}} \lambda \psi_{M_{x}}^{2}+2 \frac{M_{x}}{M_{y}} \lambda \psi_{M_{y x}}}\right. \\
& \left.\quad+\frac{\theta_{1}}{\theta_{2}}\right)>0 .
\end{aligned}
$$

Condition (v): By Eqs. (13) and (32), we have: 


$$
M S E\left(\widehat{M}_{S S 2}\right)_{\min }-M S E\left(\widehat{M}_{\text {Prop }}\right)_{\min }>0
$$

if:

$$
M_{y}^{2}\left(\frac{\lambda \psi_{M_{y}}^{2}\left(1-\rho_{y x}^{2}\right)}{1+\lambda \psi_{M_{y}}^{2}\left(1-\rho_{y x}^{2}\right)}-1+\frac{\theta_{1}}{\theta_{2}}\right)>0 .
$$

Condition (vi): By Eqs. (15) and (32), we have:

$$
\operatorname{MSE}\left(\widehat{M}_{D}\right)_{\min }-\operatorname{MSE}\left(\widehat{M}_{\mathrm{Prop}}\right)_{\min }>0
$$

if:

$$
M_{y}^{2}\left(\lambda \psi_{M_{y}}^{2}\left(1-\rho_{y x}^{2}\right)-1+\frac{\theta_{1}}{\theta_{2}}\right)>0 .
$$

Condition (vii): By Eqs. (18) and (32), we will have the equation shown in Box III.

Condition (viii): By Eqs. (23) and (32), we have:

$$
\operatorname{MSE}\left(\widehat{M}_{D 1}\right)_{\min }-\operatorname{MSE}\left(\widehat{M}_{\text {Prop }}\right)_{\min }>0
$$

if:

$$
M_{y}^{2}\left(\frac{\left(1-\lambda \psi_{M_{x}}^{2}\right) \lambda \psi_{M_{y}}^{2}\left(1-\rho_{y x}^{2}\right)}{\left(1-\lambda \psi_{M_{x}}^{2}\right)+\lambda \psi_{M_{y}}^{2}\left(1-\rho_{y x}^{2}\right)}-1+\frac{\theta_{1}}{\theta_{2}}\right)>0 .
$$

Condition (ix): By Eqs. (24) and (32), we have:

$$
\operatorname{MSE}\left(\widehat{M}_{D 2}\right)_{\min }-\operatorname{MSE}\left(\widehat{M}_{P r o p}\right)_{\min }>0,
$$

if:

$$
M_{y}^{2}\left(\frac{\theta_{1}}{\theta_{2}}-\frac{1}{4} \lambda \psi_{M_{x}}^{2}-\frac{\left(1-\frac{1}{8} \lambda \psi_{M_{x}}^{2}\right)^{2}}{1+\lambda \psi_{M_{y}}^{2}\left(1-\rho_{y x}^{2}\right)}\right)>0 .
$$

Note: The proposed estimator $\hat{M}_{\text {Prop }}$ performs better when Conditions (i)-(ix) are satisfied.

\section{Numerical comparisons}

To examine the performances of the estimators, the following data sets are used for comparison:

\section{- Population 1 [25]:}

$Y$ : Number of teaching staff,

$X$ : Number of students in four different types of schools under 36 districts in Punjab province of Pakistan.

\section{- Population 2 [12]:}

$Y$ : Number of teachers,

$X$ : Number of students in 340 districts with elementary schools in 2007.

\section{- Population 3 [21]:}

$Y$ : Graduate or higher degrees in the United States in 2007,

$X$ : Graduate or higher degrees in the United States in 2006.

\section{- Population 4 [26]:}

$Y$ : Oil prices in the current week from 1996 to 2017,

$X$ : Oil prices in previous week from 1996 to 2017.

The descriptive statistics of all populations are given in Table 1.

The following expression is employed to obtain the Percent Relative Efficiencies (PREs) with respect to Gross [1] unbiased per unit estimator:

$$
\text { PRE }=\frac{V\left(\widehat{M}_{y}\right)}{M S E\left(\widehat{M}_{j}\right) \text { or } M S E\left(\widehat{M}_{j}\right)_{\min }} \times 100,
$$

where:

$$
\begin{aligned}
j= & y, R, \operatorname{Exp}, S S 1, S S 2, D, D 1, D 2, S S 3, \\
& \operatorname{Prop}_{(\alpha=0)}, \operatorname{Prop}_{(\alpha=1)} .
\end{aligned}
$$

MSEs and PREs values based on four populations are given in Table 2.

According to Table 2, the MSE of the proposed class of estimators in all four populations is minimum compared to the considered estimators. It is also observed that when using different correlations, the proposed estimators are more efficient and stable.

$$
\begin{aligned}
& M S E\left(\widehat{M}_{S S 3}\right)_{\text {min }}-M S E\left(\widehat{M}_{\text {Prop }}\right)_{\text {min }}>0, \quad \text { if: } \\
& M_{y}^{2}\left[\frac{\lambda\left(\lambda \psi_{M_{x}}^{6}+\lambda \psi_{M_{y}}^{2} \psi_{M_{x}}^{4}+2 \lambda \psi_{M_{x}}^{4} \psi_{M_{y x}}-4 \lambda \psi_{M_{y}}^{2} \psi_{M_{x}}^{2} \psi_{M_{y x}}-4 \lambda \psi_{M_{x}}^{2} \psi_{M_{y x}}^{2}+4 \lambda \psi_{M_{y}}^{2} \psi_{M_{y x}}^{2}-4 \psi_{M_{y}}^{2} \psi_{M_{x}}^{2}+4 \psi_{M_{y x}}^{2}\right)}{16 \lambda \psi_{M_{y x}}^{2}-4 \psi_{M_{x}}^{2}-8 \lambda \psi_{M_{x}}^{2} \psi_{M_{y x}}-4 \lambda \psi_{M_{y}}^{2} \psi_{M_{x}}^{2}-3 \lambda \psi_{M_{x}}^{4}}\right. \\
& \left.\quad-1+\frac{\theta_{1}}{\theta_{2}}\right]>0 .
\end{aligned}
$$


Table 1. Descriptive statistics of all populations.

\begin{tabular}{ccccc}
\hline Notation & Population $\mathbf{1}$ & Population 2 & Population 3 & Population 4 \\
\hline $\boldsymbol{N}$ & 144 & 340 & 51 & 1134 \\
$\boldsymbol{n}$ & 10 & 49 & 11 & 210 \\
$\boldsymbol{\lambda}$ & 0.02327 & 0.00436 & 0.01782 & 0.00097 \\
$\boldsymbol{M}_{\boldsymbol{y}}$ & 2023 & 178 & 25.80 & 48.55 \\
$\boldsymbol{M}_{\boldsymbol{x}}$ & 64659 & 3526 & 25.60 & 48.49 \\
$\boldsymbol{f}_{\boldsymbol{y}}\left(\boldsymbol{M}_{\boldsymbol{y}}\right)$ & 0.00024 & 0.00182 & 0.0728 & 0.0075349 \\
$\boldsymbol{f}_{\boldsymbol{x}}\left(\boldsymbol{M}_{\boldsymbol{x}}\right)$ & 0.00001 & 0.00800 & 0.1080 & 0.0075437 \\
$\boldsymbol{\rho}_{\boldsymbol{y} \boldsymbol{x}}$ & 0.86110 & 0.92000 & 0.9956 & 0.9953 \\
$\boldsymbol{\psi}_{\boldsymbol{M}_{\boldsymbol{y}}}$ & 2.05965 & 3.08680 & 0.53242 & 2.73359 \\
$\boldsymbol{\psi}_{\boldsymbol{M}_{\boldsymbol{x}}}$ & 1.54658 & 0.03545 & 0.36168 & 2.73378 \\
$\boldsymbol{\psi}_{\boldsymbol{M}_{\boldsymbol{y} \boldsymbol{x}}}$ & 2.74295 & 0.10067 & 0.19172 & 7.43791 \\
\hline
\end{tabular}

Table 2. Mean Square Error (MSE) and Percent Relative Efficiencies (PRE) values of all considered and proposed estimators.

\begin{tabular}{|c|c|c|c|c|c|c|c|c|}
\hline \multirow[b]{2}{*}{ Estimator } & \multicolumn{2}{|c|}{ Population 1} & \multicolumn{2}{|c|}{ Population 2} & \multicolumn{2}{|c|}{ Population 3} & \multicolumn{2}{|c|}{ Population 4} \\
\hline & MSE & $P R E$ & MSE & $P R E$ & MSE & $P R E$ & MSE & $P R E$ \\
\hline$\widehat{M}_{y}$ & 403886.959 & 100.000 & 1318.303 & 100.000 & 3.36337 & 100.000 & 17.08538 & 100.000 \\
\hline$\widehat{M}_{R}$ & 109312.950 & 369.478 & 1290.618 & 102.145 & 0.36594 & 919.111 & 0.1606138 & 10637.548 \\
\hline$\widehat{M}_{\mathbf{E x p}}$ & 199667.997 & 202.279 & 1304.417 & 101.065 & 1.47661 & 227.777 & 4.35105 & 392.672 \\
\hline$\widehat{M}_{S S 1}$ & 102203.340 & 395.179 & 1242.712 & 106.083 & 3.31657 & 101.107 & 16.60673 & 102.883 \\
\hline$\widehat{M}_{S S 2}$ & 174090.410 & 231.999 & 201.205 & 655.203 & 0.02953 & 11389.197 & 0.16022 & 10664.082 \\
\hline$\widehat{M}_{D}$ & 181824.980 & 222.129 & 202.492 & 651.042 & 0.02953 & 11388.691 & 0.16023 & 10663.357 \\
\hline$\widehat{M}_{D 1}$ & 173655.141 & 232.579 & 201.205 & 655.203 & 0.02953 & 11389.198 & 0.16021 & 10664.087 \\
\hline$\widehat{M}_{D 2}$ & 171479.031 & 235.532 & 201.205 & 655.203 & 0.02946 & 11417.774 & 0.15798 & 10814.411 \\
\hline$\widehat{M}_{S S 3}$ & 103796.950 & 389.113 & 173.7406 & 758.776 & 0.03060 & 11440.852 & 0.16019 & 10665.607 \\
\hline$\widehat{M}_{\text {Prop }_{(\alpha=0)}}$ & 100940.420 & 400.124 & 173.066 & 761.732 & 0.01815 & 18528.773 & 0.11400 & 14986.947 \\
\hline$\widehat{M}_{\text {Prop }_{(\alpha=1)}}$ & 99054.183 & 407.744 & 172.717 & 763.273 & 0.02924 & 11501.591 & 0.141623 & 12064.049 \\
\hline
\end{tabular}

\section{Simulation study}

The simulation study was carried out to evaluate the performance of the estimators both empirically and theoretically. To this end, three finite populations of size 1500 generated from a skewed multivariate normal distribution with the same theoretical means of $[Y, X]$ as $\mu=[5,5]$ were taken into account and different variance covariance matrices were used, as shown in the following:

\section{Population 1:}

$$
\Sigma=\left[\begin{array}{cc}
10 & 3 \\
3 & 2
\end{array}\right]
$$

\section{Population 2:}

$$
\Sigma=\left[\begin{array}{ll}
6 & 3 \\
3 & 2
\end{array}\right] .
$$

\section{Population 3:}

$$
\Sigma=\left[\begin{array}{cc}
9 & 1.9 \\
1.9 & 4
\end{array}\right]
$$

The sample sizes $n=50,100,150,300$ were attributed to each population, and the probability density functions $f_{y}\left(M_{y}\right)$ and $f_{x}\left(M_{x}\right)$ were calculated using normal distribution. Table 3 computes the empirical and theoretical MSEs and PREs. Then, the empirical MSE was estimated using 5000 samples of different sizes selected from each population.

Based on the analysis of the simulation results of all the three artificial populations in Table 3 , it can be concluded that the proposed class of estimators 
Table 3. Empirical and theoretical Mean Square Error (MSE) and Percent Relative Efficiencies (PRE) values of different estimators with respect to $\hat{M}_{y}$.

\begin{tabular}{|c|c|c|c|c|c|c|c|c|c|c|}
\hline \multirow{3}{*}{$n$} & \multirow{3}{*}{ Estimator } & \multicolumn{3}{|c|}{ Population 1} & \multicolumn{3}{|c|}{ Population 2} & \multicolumn{3}{|c|}{ Population 3} \\
\hline & & \multicolumn{2}{|c|}{ MSE } & \multirow[b]{2}{*}{ PRE } & \multicolumn{2}{|c|}{ MSE } & \multirow[b]{2}{*}{ PRE } & \multicolumn{2}{|c|}{ MSE } & \multirow[b]{2}{*}{ PRE } \\
\hline & & Empirical & Theoretical & & Empirical & Theoretical & & Empirical & Theoretical & \\
\hline \multirow{11}{*}{50} & $\hat{M}_{y}$ & 0.306148 & 0.323727 & 100.00 & 0.183689 & 0.194236 & 100.00 & 0.275533 & 0.291354 & 100.00 \\
\hline & $\hat{M}_{R}$ & 0.236473 & 0.244817 & 132.23 & 0.097730 & 0.107067 & 181.41 & 0.327556 & 0.341346 & 85.35 \\
\hline & $\hat{M}_{\operatorname{Exp}}$ & 0.255370 & 0.267997 & 120.79 & 0.123801 & 0.134467 & 144.45 & 0.269551 & 0.283504 & 102.77 \\
\hline & $\hat{M}_{S S 1}$ & 0.318258 & 0.318130 & 101.76 & 0.188586 & 0.191566 & 101.39 & 0.285143 & 0.287387 & 101.38 \\
\hline & $\hat{M}_{S S 2}$ & 0.242741 & 0.241913 & 133.82 & 0.099727 & 0.104712 & 185.50 & 0.274293 & 0.275908 & 105.60 \\
\hline & $\hat{M}_{D}$ & 0.238789 & 0.244085 & 132.63 & 0.098889 & 0.105124 & 184.77 & 0.267575 & 0.278749 & 104.52 \\
\hline & $\hat{M}_{D 1}$ & 0.302483 & 0.241908 & 133.82 & 0.161117 & 0.104711 & 185.50 & 0.390574 & 0.275894 & 105.60 \\
\hline & $\hat{M}_{D 2}$ & 0.258115 & 0.241766 & 133.90 & 0.114109 & 0.104646 & 185.61 & 0.302462 & 0.275563 & 105.73 \\
\hline & $\hat{M}_{S S 3}$ & 0.296073 & 0.242102 & 133.71 & 0.131809 & 0.104845 & 185.26 & 0.411307 & 0.275573 & 105.73 \\
\hline & $\hat{M}_{\operatorname{Prop}_{(\alpha=0)}}$ & 0.330339 & 0.241733 & 133.92 & 0.194626 & 0.104634 & 185.63 & 0.413497 & 0.275120 & 105.90 \\
\hline & $\hat{M}_{\operatorname{Prop}(\alpha=1)}$ & 0.252095 & 0.241607 & 133.99 & 0.108878 & 0.104620 & 185.66 & 0.277523 & 0.274128 & 106.28 \\
\hline \multirow{11}{*}{100} & $\hat{M}_{y}$ & 0.136593 & 0.156282 & 100.00 & 0.081956 & 0.093769 & & 0.122933 & 0.140654 & 100.00 \\
\hline & $\hat{M}_{R}$ & 0.105485 & 0.118188 & 132.23 & 0.043630 & 0.051688 & 181.41 & 0.145648 & 0.164788 & 85.35 \\
\hline & $\hat{M}_{\operatorname{Exp}}$ & 0.113646 & 0.129378 & 120.79 & 0.054098 & 0.064915 & 144.45 & 0.119849 & 0.136864 & 102.77 \\
\hline & $\hat{M}_{S S 1}$ & 0.138803 & 0.154965 & 100.85 & 0.082791 & 0.093142 & 100.67 & 0.124701 & 0.139721 & 100.67 \\
\hline & $\hat{M}_{S S 2}$ & 0.106430 & 0.117325 & 133.20 & 0.045065 & 0.050953 & 184.03 & 0.119171 & 0.133903 & 105.04 \\
\hline & $\hat{M}_{D}$ & 0.106030 & 0.117834 & 132.63 & 0.044935 & 0.050750 & 184.77 & 0.118115 & 0.134568 & 104.52 \\
\hline & $\hat{M}_{D 1}$ & 0.132564 & 0.117325 & 133.20 & 0.070592 & 0.050853 & 184.39 & 0.173543 & 0.133901 & 105.04 \\
\hline & $\hat{M}_{D 2}$ & 0.112452 & 0.117291 & 133.24 & 0.049209 & 0.050838 & 184.45 & 0.132336 & 0.133822 & 105.10 \\
\hline & $\hat{M}_{S S 3}$ & 0.122492 & 0.117370 & 133.15 & 0.099777 & 0.050795 & 184.60 & 0.134908 & 0.133784 & 105.13 \\
\hline & $\hat{M}_{\operatorname{Prop}_{(\alpha=0)}}$ & 0.143380 & 0.117368 & 133.16 & 0.092437 & 0.050735 & 184.82 & 0.179402 & 0.133717 & 105.19 \\
\hline & $\hat{M}_{\operatorname{Prop}_{(\alpha=1)}}$ & 0.108088 & 0.117254 & 133.29 & 0.046540 & 0.050632 & 185.20 & 0.119465 & 0.133484 & 105.37 \\
\hline \multirow{11}{*}{150} & $\hat{M}_{y}$ & 0.087658 & 0.100467 & 100.00 & 0.052595 & .060280 & 100.00 & 8892 & 0.090420 & 100.00 \\
\hline & $\hat{M}_{R}$ & 0.068742 & 0.075978 & 132.23 & 0.028247 & 0.033228 & 181.41 & 0.093848 & 0.105935 & 85.35 \\
\hline & $\hat{M}_{\mathrm{Exp}}$ & 0.073758 & 0.083172 & 120.79 & 0.034606 & 0.041731 & 144.45 & 0.077276 & 0.087984 & 102.77 \\
\hline & $\hat{M}_{S S 1}$ & 0.088259 & 0.099921 & 100.55 & 0.052769 & 0.060020 & 100.43 & 0.079355 & 0.090033 & 100.43 \\
\hline & $\hat{M}_{S S 2}$ & 0.068951 & 0.075540 & 133.00 & 0.029302 & 0.032585 & 184.99 & 0.076545 & 0.086233 & 104.86 \\
\hline & $\hat{M}_{D}$ & 0.069058 & 0.075750 & 132.63 & 0.029298 & 0.032625 & 184.77 & 0.076309 & 0.086508 & 104.52 \\
\hline & $\hat{M}_{D 1}$ & 0.085043 & 0.075540 & 133.00 & 0.045133 & 0.032585 & 184.99 & 0.110684 & 0.086232 & 104.86 \\
\hline & $\hat{M}_{D 2}$ & 0.072697 & 0.075526 & 133.02 & 0.031426 & 0.032579 & 185.00 & 0.084669 & 0.086199 & 104.90 \\
\hline & $\hat{M}_{S S 3}$ & 0.107018 & 0.075559 & 132.97 & 0.048483 & 0.032598 & 184.92 & 0.089474 & 0.086899 & 104.06 \\
\hline & $\hat{M}_{\operatorname{Prop}_{(\alpha=0)}}$ & 0.089938 & 0.075510 & 133.05 & 0.061190 & 0.032576 & 185.04 & 0.114622 & 0.086156 & 104.95 \\
\hline & $\hat{M}_{\operatorname{Prop}_{(\alpha=1)}}$ & 0.069556 & 0.075001 & 133.95 & 0.029932 & 0.032147 & 187.51 & 0.076447 & 0.086059 & 105.07 \\
\hline \multirow{11}{*}{300} & $\hat{M}_{y}$ & 0.037044 & 0.044652 & 100.00 & 0.022226 & 0.026791 & 100.00 & 0.033340 & 0.040187 & 100.00 \\
\hline & $\hat{M}_{R}$ & 0.029300 & 0.033768 & 132.23 & 0.011986 & 0.014768 & 181.41 & 0.040547 & 0.047082 & 85.35 \\
\hline & $\hat{M}_{\operatorname{Exp}}$ & 0.031229 & 0.036965 & 120.79 & 0.014392 & 0.018547 & 144.45 & 0.032560 & 0.039104 & 102.77 \\
\hline & $\hat{M}_{S S 1}$ & 0.037163 & 0.044544 & 100.24 & 0.022263 & 0.026740 & 100.19 & 0.033432 & 0.040110 & 100.19 \\
\hline & $\hat{M}_{S S 2}$ & 0.029297 & 0.033625 & 132.79 & 0.012613 & 0.014492 & 184.87 & 0.032058 & 0.038394 & 104.67 \\
\hline & $\hat{M}_{D}$ & 0.029387 & 0.033667 & 132.63 & 0.012624 & 0.014500 & 184.77 & 0.032029 & 0.038448 & 104.52 \\
\hline & $\hat{M}_{D 1}$ & 0.035808 & 0.033625 & 132.79 & 0.018890 & 0.014492 & 184.87 & 0.048751 & 0.038394 & 104.67 \\
\hline & $\hat{M}_{D 2}$ & 0.030652 & 0.033622 & 132.80 & 0.013044 & 0.014491 & 184.88 & 0.036065 & 0.038387 & 104.69 \\
\hline & $\hat{M}_{S S 3}$ & 0.039510 & 0.033629 & 132.78 & 0.027763 & 0.014495 & 184.84 & 0.041918 & 0.038381 & 104.70 \\
\hline & $\hat{M}_{\operatorname{Prop}_{(\alpha=0)}}$ & 0.038825 & 0.033642 & 132.73 & 0.028340 & 0.014490 & 184.89 & 0.050344 & 0.038378 & 104.71 \\
\hline & $\hat{M}_{\operatorname{Prop}_{(\alpha=1)}}$ & 0.029498 & 0.033619 & 132.82 & 0.012789 & 0.014423 & 185.75 & 0.032083 & 0.038359 & 104.76 \\
\hline
\end{tabular}


performs more efficiently, thus including less MSE than all the other considered estimators in all populations. Of note, the empirical MSE of $\widehat{M}_{S S 3}$ is very high in all populations, hence performing quite poorly.

\section{Conclusion}

The current study proposed a new class of ratio-cumproduct type exponential estimators for the population median $\left(M_{y}\right)$ under simple random sampling using the supplementary variable. The bias and Mean Square Error (MSE) expressions were obtained up to first order of approximation. Theoretical conditions were derived under which the proposed class of estimators performed better. Tables 2 and 3 compared the proposed classes of estimators $\widehat{M}_{\mathrm{Prop}_{(\alpha=0)}}$ and $\widehat{M}_{\mathrm{Prop}_{(\alpha=1)}}$ with the unbiased estimator of median and other considered estimators both theoretically and empirically in terms of MSEs and Percent Relative Efficiencies (PREs) using different sample sizes. It was observed that the proposed class of estimators had minimum MSE; hence, it performed better in all the four populations. A simulation study reflected the same behavior in all populations. Therefore, the practical application of the proposed estimators $\widehat{M}_{\operatorname{Prop}_{(\alpha=0)}}$ and $\widehat{M}_{\operatorname{Prop}_{(\alpha=1)}}$ in different fields of engineering and data analysis is highly recommended.

\section{Acknowledgments}

Authors are thankful to the editor and anonymous referees for their valuable suggestions and comments which helped improve the quality of this research.

\section{References}

1. Gross, S.T., Median Estimation in Sample Surveys, Survey Research Method Section, American Statistical Association, pp. 181-184 (1980).

2. Kuk, Y.C.A. and Mak, T.K. "Median estimation in the presence of auxiliary information", Journal of Royal Statistical Society, B, 51, pp. 261-269 (1989).

3. Singh, S., Joarder, A.H., and Tracy, D.S. "Median estimation using double sampling", Australian and Newzeland Journal of Statistics, 43(1), pp. 33-46 (2001).

4. Singh, H.P., Singh, S., and Joarder, A.H. "Estimation of population median when mode of an auxiliary variable is known", J. Statist. Res., 37(1), pp. 57-63 (2003).

5. Singh, H.P., Sidhu, S.S., and Singh, S. "Median estimation with known interquartile range of auxiliary variable", Int. J. Appl. Math. Statist., 4, pp. 68-80 (2006).

6. Singh, S., Singh, H.P., Upadhyaya, L.N. "Chain ratio and regression type estimators for median estimation in survey sampling", Statistical Papers, 48(1), pp. 2346 (2007).

7. Gupta, S., Shabbir, J., and Ahmad, S. "Estimation of median in two phase sampling using two auxiliary variables", Communications in Statistics-Theory and Methods, 37(11), pp. 1815-1822 (2008).

8. Aladag, S. and Cingi, H. "New estimators for the population median in simple random sampling", Proceedings of the Tenth Islamic Countries Conference on Statistical Sciences (ICCS-X), Held in New Cairo, Egypt, pp. 375-383 (2009).

9. Singh, H.P. and Solanki R.S. "Some classes of estimators for the population median using auxiliary information", Communications in Statistics-Theory and Methods, 42, pp. 4222-4238 (2013).

10. Jhajj, H.S., Kaur, H., and Walia, G. "Efficient family of ratio-product type estimators of median", Model Assisted Statistics and Applications, 9, pp. 277-282 (2014).

11. Sharma, P. and Singh, R. "Generalized class of estimators for population median using auxiliary information", Hacettepe Journal of Mathematics and Statistics, 44(2), pp. 443-453 (2015).

12. Aladag, S. and Cingi, H. "Improvement in estimating the population median in simple random sampling and stratified random sampling using auxiliary information", Communications in Statistics-Theory and Methods, 44, pp. 1013-1032 (2015).

13. Shabbir, J. and Gupta, S. "Improved estimation of finite population median under two-phase sampling when using two auxiliary variables", Scientia Iranica, 22(3), pp. 1271-1277 (2015).

14. Shabbir, J. and Gupta, S. "A generalized class of difference-type estimator for population median in survey sampling", Hacettepe Journal of Mathematics and Statistics, 46(5), pp. 1015-1028 (2017).

15. Solanki, R.S. and Singh, H.P. "Some classes of estimators for median estimation in survey sampling", Communications in Statistics-Theory and Methods, 44(7), pp. 1450-1465 (2015).

16. Enang, E.I., Etuk, S.I., Ekpenyong, E.J., and Akpan, V.M. "An alternative exponential estimator of population median", International Journal of Statistics and Economics, 17(3), pp. 85-97 (2016).

17. Jhajj, H.S., Kaur, H., and Jhajj, P. "Efficient family of estimators of median using twophase sampling design", Communications in Statistics-Theory and Methods, 45(15), pp. 4325-31 (2016).

18. Koyuncu, N. "Improved ratio estimation of population mean under median and neoteric ranked set sampling", AIP Conference Proceedings, AIP Publishing, 1863(1), pp. 1200-1204 (2017). 
19. Irfan, M., Javed, M., and Lin, Z. "Efficient ratiotype estimators of finite population mean based on correlation coefficient", Scientia Iranica, 25(4), pp. 2361-2372 (2018).

20. Yadav, D.K., Shukla, A.K., Tomer, S., and Kumar, B. "Predictive estimation of finite population mean using coefficient of kurtosis and median of an auxiliary variable under simple random sampling scheme", International Journal of Mathematical Archive EISSN 2229-5046, 9(5), pp. 137-43 (2018).

21. Bandyopadhyay, A., Singh, G.N., and Das, S. "Estimation of population median in presence of non-response under two-phase sampling", Sri Lankan Journal of Applied Statistics, 17(2), pp. 112-133 (2018).

22. Javed, M., Irfan, M., and Pang, T. "Hartley-Ross type unbiased estimators of population mean Using two auxiliary variables", Scientia Iranica, E, 26(6), pp. 3835-3845 (2019). DOI: 10.24200/SCI.2018.5648.1397

23. Baig, A., Masood, S., and Tarray, T.A. "Improved class of difference-type estimators for population median in survey sampling", Communications in Statistics-Theory and Methods, 49(23), pp. 5778-5793 (2019). DOI: 10.1080/03610926.2019.1622017

24. Muneer, S., Khalil, A., Shabbir, J., and Narjis, G. "A new improved ratio-product type exponential estimator of finite population variance using auxiliary information", Journal of Statistical Computation and Simulation, 88(16), pp. 3179-3192 (2018).

25. "PDS Punjab Development Statistics", Bureau of Statistics, Government of the Punjab, Lahore, Pakistan, pp. 114-116 (2012).

26. Amir, M., Shabri, A., and Ishaq, M. "Improving forecasting accuracy of crude oil price using decomposition ensemble model with reconstruction of IMFs based on ARIMA model", Malaysian Journal of Fundamental and Applied Sciences, 14(4), pp. 471-483 (2018).

\section{Biographies}

Siraj Muneer is a $\mathrm{PhD}$ scholar at the Department of Statistics, University of Peshawar, Pakistan. He has published many articles in internationally reputed journals in the field of survey methodologies and data analysis. His area of interest includes sampling theory and probability.

Alamgir Khalil is an Associate Professor of Statistics at the Department of Statistics University of Peshawar, Pakistan. He received his $\mathrm{PhD}$ in Statistics from University of Peshawar in 2013. He has published many papers in national and international reputed journals in the field of probability and distribution theory. His area of interest includes sampling theory and probability.

Javid Shabbir is working as a Tenured Professor of Statistics at the Department of Statistics Quaid-iAzam University Islamabad, Pakistan. He received his $\mathrm{PhD}$ in Statistics from Kent University at Canterbury, UK in 1997. He obtained Post-Doctoral positions at University of Southern Maine, USA in 2003 and University of North Carolina at Greensboro USA in 2005. He has published about 300 papers in different internationally reputed journals. His area of research includes survey sampling and randomized response techniques.

Ghulam Narjis received her $\mathrm{PhD}$ degree in Statistics from Quaid-iAzam University Islamabad, Pakistan. She has published many articles in internationally reputed journals in the field of survey methodologies, randomized response, and data analysis. Her area of interest includes sampling theory and Bayesian analysis. 